Psychological Medicine, 1997, 27, 253-260. Copyright (C) 1997 Cambridge University Press

\title{
EDITORIAL
}

\section{Postpartum depression and child development ${ }^{1}$}

Depression has a profound impact on parameters of interpersonal behaviour (Lewinsohn et al. 1970; Libert \& Lewinsohn, 1973; Teasdale et al. 1980). Studies of healthy mother-infant dyads have demonstrated that infants are highly sensitive to their interpersonal environment (Murray, 1988). It is, therefore, likely that postpartum depression, by virtue of its impact on maternal interpersonal functioning, will disrupt normal infant engagements with the mother and, as a consequence, impair infant developmental progress. In recent years a considerable body of research surrounding this question has accumulated.

\section{POSTPARTUM DEPRESSION AND EARLY INFANCY}

A number of studies have been conducted that examine the behaviour of infants of depressed mothers in the context of face-to-face interactions, principally with the mother. In a series of investigations Field and colleagues have found the behaviour of infants of 3 to 7 months old to be less optimal where the mother is depressed. Thus, Field (1984) observed depressed and nondepressed mothers in interaction with their infants at 3 months postpartum, and found the depressed mothers' infants to show fewer positive facial expressions and more negative expressions and protest behaviour. Similarly, Field and colleagues (1985) reported that, compared with controls, the 4-month-old infants of 12 mothers with pregnancy problems (most of whom had depressed mood postpartum) were more drowsy and fussy, and less relaxed and contented during a 10 min interaction. In a further study conducted by this group (Field et al. 1988) infants of mothers with depressed mood and infants of non-depressed controls were compared at 3 to 6 months postpartum in interaction both with their mothers and with non-depressed unfamiliar adults; and in both conditions the infants of depressed women were rated worse on measures of state, physical activity, facial expressions, vocalizations and fussing.

The general finding of less optimal infant behaviour in the infants of depressed mothers has been confirmed in a report by Cohn and colleagues (1986). They studied a high risk sample of mothers with depressive disorder who had been referred to a family intervention service. They found that the behaviour during face-to-face interactions of the 6 to 7 -month infants of these women was characterized by high levels of withdrawn behaviour and an absence of positive affect. A notable feature of this study was the identification of different profiles of disturbed infant behaviour, some infants showing a predominance of avoidance, while others showed high rates of protest behaviour. This latter finding was confirmed in a further study by Field and colleagues (1990).

The studies summarized above, which consistently revealed deficits in the interactions of infants of depressed mothers, were generally conducted on socio-economically disadvantaged populations. For example, in the study by Field and colleagues (1990), 35\% of the mothers were 'adolescent' and $75 \%$ were single mothers. In contrast with the findings of these reports, two recent studies of mothers and infants recruited from low risk samples have failed to identify clearly different profiles of infant behaviour in depressed and well groups. Campbell et al. (1995) investigated mother-infant interactions in a community sample of primiparous, White, married mothers aged between 18 and 35 years of age, with full-term, healthy singleton infants. Observations of face-to-face, toy play and

1 Address for correspondence: Professor Lynne Murray, University of Reading, Department of Psychology, 3 Early Gate, Whiteknights, Reading RG6 6AL. 
feeding interactions were made at 2, 4 and 6 months postpartum and the data examined in terms of the presence or absence of maternal depression at 2 months postpartum. No differences were found between the two samples of infants on any of the measures of infant engagement. However, when chronicity of depression was taken into account, infants of mothers whose depression persisted to 6 months postpartum were found to be less positive in their early toy play interactions than were infants of women whose symptoms were subclinical or who remitted. A similar study has been conducted in Britain by Murray and colleagues (1996a) with a representative community sample of primiparous mothers of healthy infants. Women with postpartum depressive disorder were observed in face-to-face interaction with their infants at 2 months postpartum. Little difference was observed in the behaviour of the two groups of infants: levels of positive engagement with the mother were comparable, as were expressions of avoidance and distress. The only difference between the two groups of infants was in the incidence of disrupted behaviour, when the infant would momentarily arrest his or her active engagement and avoid contact.

The conclusion from the studies of the behaviour of infants in face-to-face engagement summarized above would appear to be that there is an interaction between maternal postpartum depression and indices of socio-economic adversity with respect to infant behaviour. Thus, where there is no wider social disadvantage, no association is found between postpartum depression and indices of early infant interactional disturbance; and where there is such disadvantage, the infants of women with postpartum depression are found to evince a range of disturbances in interactional behaviour.

Two studies have assessed the infants of depressed mothers independently of interactions with their mothers. Cutrona \& Troutman (1986), in a study of a sample of unselected women found an association between mothers' level of depressed mood and the presence of difficult behaviour in the infants at 3 months, as assessed by maternal reports and crying records, and by direct observations of infant crying. Similarly, Whiffen \& Gotlib (1989) assessed the 2-month-old infants of women experiencing a depressive disorder and well mothers, and found that the infants of the depressed mothers were more tense, less content and deteriorated more quickly under the stress of developmental testing.

\section{POSTPARTUM DEPRESSION AND LATER INFANCY}

A number of studies have examined the 12 to 21 month-old children of mothers who have had a postnatal depression. These studies have generally found an association between early maternal depression and adverse cognitive and emotional infant outcome.

\section{Cognitive development}

Two studies have reported on the cognitive outcome of 12 to 18 -month-old infants of mothers who had been depressed postnatally. Lyons-Ruth et al. (1986), in a comparison of mothers and infants who had been referred to an infant intervention service and matched community controls, found that increased levels of maternal depression were significantly related to poorer infant mental and motor development as assessed by the Bayley Scales (Bayley, 1969) at 1 year. This relationship still obtained after controlling for maternal IQ. Similarly, Murray (1992), in a comparison of the development of the community sample of children of mothers who had had postpartum depression with the children of well mothers, found a significant difference between the two groups of children in terms of a number of indices of cognitive development. At follow-up at 18 months of age, compared to the infants of well mothers, infants of mothers who had had postpartum depression were significantly more likely to fail on Stage V of Piaget's Object Concept Task (Piaget, 1954). The findings in relation to the infants' overall cognitive performance, as measured by the Bayley Scales of mental development, were somewhat more complicated in that there was an interaction between maternal mental state history and infant gender, boys whose mothers had had postnatal depression 
had the lowest Bayley scores whereas the girls had the highest (Murray et al. 1996a). The findings for both the Object Concept Task and the Bayley Scales still obtained when the presence of any current depression (i.e. at 18 months) was taken into account.

\section{Emotional development}

The impact of postpartum depression on the emotional development of infants has been studied in three ways. First, the quality of the infant's interpersonal functioning has been examined when the infant has been in direct communicative engagement with the mother (Stein et al. 1991). Secondly, assessments have been made of the quality of infant attachment to the mother (Lyons-Ruth et al. 1986; Murray, 1992; Teti et al. 1995; Campbell \& Cohn, 1996), by means of the Ainsworth Strange Situation Procedure (Ainsworth \& Wittig, 1969). Finally, account has been taken of the level of behavioural problems in the infants (Murray, 1992).

Stein et al. (1991) made standardized ratings of the quality of mother-infant interactions in a representative community sample of women with postpartum depression and 49 well controls together with their 19-month-old children. Compared with the children of the well mothers, the children of the mothers who had had postpartum depression showed less affective sharing, a lower rate of overall interaction, less concentration and more negative responses. They also showed less sociability to a stranger. This relationship between adverse infant outcome and postpartum depression was found still to obtain in cases where the maternal depression had remitted.

Four studies have systematically assessed the quality of infant attachment in the context of postpartum depression using standard procedures. In the study conducted by Lyons-Ruth and colleagues (1986) an association was found between high levels of depression and insecure infant attachment at 12 months. Similarly, Murray (1992) found a significant association between the occurrence of depression in the postnatal period (minor or major depression) and insecurity of attachment at 18 months, with the predominant insecure attachment profile being one of avoidance. Teti and colleagues also found that, compared with a non-depressed control group, high rates of insecurity in late infancy obtained in the offspring of clinically referred depressed mothers (Teti $e t$ al. 1995). In contrast to these three reports, however, Campbell \& Cohn (1996) found no association between attachment quality and the occurrence or duration of postpartum depression in their low risk sample, although high rates of insecurity were present in both case and control groups. Stein et al. (1991), in the study discussed above, made no formal assessment of attachment status; but did observe the children's reaction to being separated from the mother when in the presence of a stranger. They found that distress was evidenced by significantly fewer of the children in the index group, possibly indicative of a higher rate of avoidantly attached infants.

Finally, Murray (1992) interviewed mothers when their infants were 18 months old using a modified version of the Behavioural Screening Questionnaire (Richman \& Graham, 1971). She found that, compared with women who had been well in the postpartum period, women who had experienced postpartum depression were more likely to report a behavioural difficulty in the child. These difficulties most frequently consisted of problems with sleeping or eating, excessive temper tantrums and separation problems.

Taken together, these studies attest to a significantly raised level of emotional difficulties in late infancy in the children of mothers who have had a postpartum depression.

\section{POSTPARTUM DEPRESSION AND EARLY CHILDHOOD}

There have been few studies of the longer term outcome of children of mothers who have had postpartum depression. The studies that have been reported have produced somewhat less consistent findings than those obtained in infancy. 


\section{Cognitive development}

Three British studies have examined the relationship between postpartum depression and the cognitive development of 4- to 5-year-old children. Cogill et al. (1986) followed up children from a low risk sample originally recruited in early pregnancy. The children were assessed on the McCarthy Scales of Children's Abilities (McCarthy, 1972) at 4 years, and the average score on the General Cognitive Index (GCI) of the index group (that is, where the mother had had a depressive disorder at some point in the postpartum year) was found to be significantly lower than that of the children of women who had not been depressed in the first postpartum year. Further examination of the data from this study (Hay \& Kumar, 1995) revealed that the relationship between the maternal mood disorder and compromised child cognitive development was confined to cases where the mother was less well educated. A second British sample, drawn from a high risk population, was also followed up at 4 years and similarly assessed (Sharp et al. 1995). Comprehensive data were available for 60 children whose mothers had been depressed during the first postpartum year and 75 children of well controls. As in the study of Cogill et al. the mean GCI was significantly lower for the children of mothers who had been depressed postnatally than that for the control children. This relationship between maternal mood disorder and poor cognitive status was confined to the boys of postnatally depressed women whose scores were, on average, one standard deviation lower than those for the boys of control group mothers. As in Murray's study of cognitive functioning in late infancy, however, the girls of postnatally depressed women performed well. In both London studies the relationship between postpartum depression and adverse child cognitive outcome was still apparent when depression occurring beyond the first postpartum year had been taken into account.

The findings of the two studies summarized above stand in contrast to those of Murray and colleagues (1996b). In this prospective, longitudinal study of a low risk sample, $95 \%$ of the cohort of postnatally depressed and well women and their children recruited at 2 months postpartum and reassessed at 18 months were followed up at 5 years. No relationship was found between postpartum depression and the children's performance on the McCarthy Scales or on Piagetian conservation tasks, even when child gender and the social class of the families had been taken into account.

\section{Emotional/social functioning}

There have been four comparative follow-up studies of British samples of women with postpartum depression and well controls that report on child emotional and/or social development. The first of these was conducted by Ghodsian et al. (1984). The mental state of a community sample of women recruited antenatally had been systematically assessed on a number of occasions up to 42 months postpartum. At the follow-up assessment the mothers were interviewed about the presence of child behaviour problems and, among the children whose mothers had been depressed at 4 months postpartum, increased levels of difficulty were found to be present. However, when later and current maternal depression were taken into account, the association between postpartum depression and childhood behaviour problems disappeared.

Wrate et al. (1985), also following up a representative community sample, found that, while women with prolonged postpartum depression showed no increase in reported child behavioural disturbance at 3 years, those who had experienced milder episodes of relatively short duration (approximately 1 month) did report raised levels of child difficulties.

Caplan et al. (1989) assessed the children in the sample followed up by Cogill et al. (1986), and interviewed the mothers when the children were 4 years old. Although there was a tendency for levels of child disturbance to be raised where the mother had been depressed between the birth and 3 months postpartum, more important factors affecting child outcome were current maternal depression, the presence of marital conflict and a history of psychiatric disturbance in the father.

Clearly, some caution is required when considering the findings of the three studies reported since the only source of information of child disturbance was the mothers themselves. A study in which 
independent assessments of child behaviour problems and social functioning was made was carried out with the children in the prospective longitudinal study of Murray and colleagues reported above (Sinclair \& Murray, 1996). Class teachers completed a number of questionnaires concerning child adjustment when the children had attended school for one term. The children of mothers who had experienced postpartum depression were found to have increased levels of behavioural disturbance of a clinically significant nature. Such disturbance was largely confined to the boys of depressed mothers and was most apparent in the area of antisocial behaviour. On certain other measures there were significant interactions between postpartum depression and child gender. Thus, on a measure of general readiness for school, the boys of postnatally depressed mother were rated as significantly less mature than the girls of depressed mothers, who themselves were no different from boy and girl controls. The same pattern of findings obtained on an assessment of child temperament with boys in the index group rated as high on the activity and distractibility scales, whereas the girls of these mothers were, by contrast, rated similarly to control group children. Indeed, on a measure of social relationships there was a trend for the girls of mothers who had had postpartum depression to be rated as more pro-social than controls.

\section{FACTORS MEDIATING THE ASSOCIATION BETWEEN POSTPARTUM DEPRESSION AND ADVERSE CHILD OUTCOME}

The principal question arising from the findings outlined above is that of the mechanisms mediating the associations between the postpartum mood disorder and adverse infant and child development. A major conclusion to emerge from the studies conducted to date is that it is the impaired patterns of interaction occurring between mother and child in the context of depression, rather than the child's exposure to depressive symptoms per se, which is important to the child's functioning.

Impairments in the face-to-face engagements of depressed mothers have not only been found to be associated with immediate signs of behavioural disturbance in the infants, but also appear to mediate some of the longer term adverse child outcome. Thus, in the longitudinal study of Murray and colleagues, the adverse impact of postpartum depression on infant cognitive outcome at 18 months was determined by the quality of mother-infant interactions at 2 months, maternal insensitivity to the infant's experience and the failure to engage the infant in active communication, particularly marked in depressed mothers of male infants, predicted poor performance both on object permanence tasks and the more general assessment of cognitive functioning (Murray et al. 1993, 1996a). Furthermore, not only did those infants whose mothers showed insensitive behaviour in the postpartum period have more adverse cognitive outcome at 18 months, but the trajectory of their cognitive development from this point onwards was markedly constrained by their 18 month functioning (Murray et al. 1996b).

A critical issue arising from the association between early mother-infant interactions and the longer term functioning of the child is whether this indicates a sensitive period in infant development, with early infant exposure to the impairments in maternal interactions being sufficient to explain later adverse outcome. An alternative to this explanation is that there are enduring features of maternal engagement with the child (possibly initiated by the affective disorder) that account for the longer term child deficits. There is evidence for some degree of continuity in the style of mothers' communication with their offspring, despite remission from depression (Weissman \& Paykel, 1974; Stein et al. 1991; Murray et al. 1996 b). Nevertheless, data from the longitudinal study of Murray and colleagues (1996b) indicate that the infant's experience of impairments in maternal communication early on may be more important than later exposure in determining cognitive outcome. Thus, in the 5-year-old children, although current maternal communication was indeed found to be associated with cognitive performance, once the child's functioning at 18 months postpartum (itself predicted by the 2 month interactions) was taken into account, the influence of the current style of maternal behaviour on child cognitive functioning was no longer significant. While this is consistent with a sensitive period account, the detailed nature of the processes that may be involved remains unclear. 
An issue that remains to be resolved concerns the extent to which any continuing impairments in maternal interactions are themselves maintained by infant behaviour. It is evident from a great deal of research with normal populations that, whatever their initial input, infants rapidly contribute actively to interactions with others. The finding of Field and colleagues (1988) that the avoidance and unresponsiveness of the infants of depressed mothers causes non-depressed adults to behave in a depressed-like manner when interacting with them, suggests that the infant may indeed make a significant contribution to the persistence of difficulties in maternal communication in the context of postpartum depression. This finding raises the more general question of the possible contribution of infant variables to the developmental process. It may be the case, for example, that individual infant characteristics influence the occurrence of maternal depression itself, the quality of mother-infant interactions, and even subsequent child development. The cross-sectional studies of Cutrona \& Troutman (1986) and Whiffen \& Gotlib (1989), which found difficult infant behaviour at 2 to 3 months to be associated with the mood disorder, are certainly consistent with there being an impact on maternal mental state of infant characteristics; but the direction of effects is unclear. A prospective study conducted by Murray and colleagues (1996c), however, did reveal a significant impact of infant variables on the occurrence of maternal depression. The presence of either poor motor control or irritable behaviour in a normal sample of healthy full-term neonates of well mothers was found to increase the risk of subsequent postpartum depression by a factor of approximately four. However, although difficult neonatal behaviour did appear to precipitate the onset of maternal depression, there was no evidence that differences in infant functioning in the neonatal period influenced the quality of maternal face-to-face interactions with the infant at 2 months. By this time, the mother's mental state, together with her social circumstances, were the overriding influences on the quality of mother-infant engagements. In addition, with regard to the longer term development of the child, there was no evidence that child outcome could be explained by individual infant differences. Thus, infant behavioural disturbance at 18 months showed no association with neonatal characteristics, but was significantly predicted by postnatal depression (Murray \& Cooper, 1996). In addition, compared with the infants of women with postpartum depression who received no treatment, the infants of those who received a brief psychological intervention between 2 and 4 months postpartum were found to have a significantly lower rate of behaviour problems at 18 months (Cooper \& Murray, 1996). With regard to cognitive outcome, the finding that the infant's behaviour during interactions with the mother at two months was significantly predictive of later cognitive performance, whereas infant behaviour during an interaction with a researcher was not, also indicates the primary role of maternal rather than infant factors.

The evidence outlined above regarding the mechanisms mediating child development in the context of postpartum depression concerns proximal influences; that is, interactions impinging directly on child experience. A wider issue arises, however, in relation to the more distal environmental factors influencing mother-infant interactions and child outcome. As will be evident from the summary of the research findings, variability in outcome is, in part, related to differences in the nature of the samples studied. Samples drawn from disadvantaged populations evidenced, in the context of maternal depression, both more severe disturbance in interactions (Field et al. 1988, 1990) and more adverse child cognitive functioning (Sharp et al. 1995) than those from low risk groups (Campbell et al. 1995; Murray et al. 1996a,b), suggesting the synergistic influences of depression and social disadvantage.

\section{CONCLUSION}

There is an accumulating body of evidence implicating postpartum depression in a range of adverse child cognitive and emotional outcomes, particularly in the boys of depressed mothers. While infant factors do appear to contribute to the inception of the maternal depression, the balance of evidence strongly suggests that it is the maternal interactional style, secondary to the affective disorder and/or social adversity, which is the potent causative agent. Observational and experimental studies 
are needed to elucidate the process by which maternal behaviour becomes disturbed. In addition, the role of the infant in the development and maintenance of the impaired interactions needs to be clarified, as does the basis for the distinctive trajectories of male and female children. The idea of there being a sensitive developmental period has received some support, at least with respect to cognitive development. However, little is known of the precise parameters involved. The explanatory potential of treatment studies has been little exploited, and that of sibling studies not at all. These could in the future contribute much to elucidating the relative contributions of genetic and environmental factors to the association between postpartum depression and adverse child outcome.

LYNNE MURRAY AND PETER J. COOPER

Lynne Murray is supported by the Medical Research Council of Great Britain.

\section{REFERENCES}

Ainsworth, M. D. \& Wittig, B. A. (1969). Attachment and exploratory behaviour in one-year-olds in a strange situation. In Determinants of Infant Behaviour (Vol. 4) (ed. B. M. Foss), pp. 111-136. Methuen: London.

Bailey, N. (1969). Bayley Scales of Infant Development. Psychological Corporation: New York.

Campbell, S. B. \& Cohn, J. F. (1996). The timing and chronicity of postpartum depression: implications in infant development. In Postpartum Depression and Child Development (ed. L. Murray and P. J. Cooper). Guilford Press: New York. (In the press.)

Campbell, S. B., Cohn, J. F. \& Meyers, T. (1995). Depression in firsttime mothers: mother-infant interaction and depression chronicity. Developmental Psychology 31, 349-357.

Caplan, H., Cogill, S., Alexandra, H., Robson, K., Katz, R. \& Kumar, R. (1989). Maternal depression and the emotional development of the child. British Journal of Psychiatry 154, 818-823.

Cogill, S., Caplan, H., Alexandra, H., Robson, K. \& Kumar, R. (1986). Impact of postnatal depression on cognitive development in young children. British Medical Journal 292, 1165-1167.

Cohn, J. F., Matias, R., Tronick, E. Z., Connell, D. \& Lyons-Ruth, D. (1986). Face-to-face interactions of depressed mothers and their infants. In Maternal Depression and Infant Disturbance. New Directions for Child Development, No. 34 (ed. E. Z. Tronick and T. Field), pp. 31-45. Jossey Bass: San Francisco.

Cooper, P. J. \& Murray, L. (1996). The impact of postnatal depression on infant development: a treatment trial. In Postpartum Depression and Child Development (ed. L. Murray and P. J. Cooper). Guilford Press: New York. (In the press.)

Cutrona, C. E. \& Troutman, B. R. (1986). Social support, infant temperament, and parenting self-efficacy: a mediational model of postpartum depression. Child Development 57, 1507-1518.

Field, T. (1984). Early interactions between infants and their postpartum depressed mothers. Infant Behaviour and Development 7, 517-522.

Field, T., Sandberg, D., Garcia, R., Vega-Lahr, N., Goldstein, S. \& Guy, L. (1985). Pregnancy problems, postpartum depression and early mother-infant interactions. Developmental Psychology 21, 1152-1156.

Field, T., Healy, B., Goldstein, S., Perry, S., Bendell, D., Schanberg, S., Zimmerman, E. A. \& Kuhn, C. (1988). Infants of depressed mothers show 'depressed' behaviour even with non-depressed adults. Child Development 59, 1569-1579.

Field, T., Healy, B., Goldstein, S. \& Guthertz, M. (1990). Behaviorstate matching and synchrony in mother-infant interactions in nondepressed versus depressed dyads. Developmental Psychology 26, 7-14.

Ghodsian, M., Zajicek, E. \& Wolkind, S. (1984). A longitudinal study of maternal depression and child behaviour problems. Journal of Child Psychology and Psychiatry 25, 91-109.
Hay, D. F. \& Kumar, R. (1995). Interpreting the effects of mothers' postnatal depression on children's intelligence: a critique and reanalysis. Child Psychiatry and Human Development 25, 165-181.

Lewinsohn, P. M., Weinstein, M. S. \& Alper, T. (1970). A behavioural approach to the group treatment of depressed persons: methodological contribution. Journal of Clinical Psychology 26, 525-532.

Libert, J. \& Lewinsohn, P. M. (1973). The concept of social skill with special reference to the behaviour of depressed persons. Journal of Consulting and Clinical Psychology 40, 304-312.

Lyons-Ruth, K., Zoll, D., Connell, D. \& Grunebaum, H. U. (1986). The depressed mother and her one-year-old infant: environment, interaction, attachment and infant development. In Maternal Depression and Infant Disturbance. New Directions for Child Development, No. 34. (ed. E. Z. Tronick and T. Field), pp. 61-82. Jossey-Bass: San Francisco.

McCarthy, D. (1972). McCarthy Scales of Children's Abilities. Psychological Corporation: New York.

Murray, L. (1988). Effects of postnatal depression on infant development: direct studies of early mother-infant interactions. In Motherhood and Mental Illness, Vol II (ed. R. Kumar and I. Brockington), pp. 159-190. John Wright: London.

Murray, L. (1992). The impact of postnatal depression on infant development. Journal of Child Psychology and Psychiatry 33, 543-561.

Murray, L. \& Cooper, P. (1996). The role of infant and maternal factors in postpartum depression, mother-infant interactions and infant outcome. In Postpartum Depression ad Child Development (ed. L. Murray and P. J. Cooper). Guilford Press: New York. (In the press.)

Murray, L., Kempton, C., Woolgar, M. \& Hooper, R. (1993). Depressed mothers' speech to their infants and its relation to infant gender and cognitive development. Journal of Child Psychology and Psychiatry 34, 1083-1101.

Murray, L., Fiori-Cowley, A., Hooper, R. \& Cooper, P. J. (1996a). The impact of postnatal depression and associated adversity on early mother infant interactions and later infant outcome. Child Development 67, 2512-2516.

Murray, L., Hipwell, A., Hooper, R., Stein, A. \& Cooper, P. J. $(1996 b)$. The cognitive development of five-year-old children of postnatally depressed mothers. Journal of Child Psychology and Psychiatry 37, 927-936.

Murray, L., Stanley, C., Hooper, R., King, F. \& Fiori-Cowley, A. $(1996 c)$. The role of infant factors in postnatal depression and mother-infant interactions. Developmental Medicine and Child Neurology 38, 109-119.

Piaget, J. (1954). The Construction of Reality in the Child. Basic Books: New York.

Richman, N. \& Graham, P. (1971). A behavioural screening questionnaire for use with three-year-old children: preliminary findings. Journal of Child Psychology and Psychiatry 12, 5-33.

Sharp, D., Hay, D., Pawlby, S., Schmucher, G., Allen, H. \& Kumar, R. (1995). The impact of postnatal depression on boys intellectual 
development. Journal of Child Psychology and Psychiatry 36, 1315-1337.

Sinclair, D. A. \& Murray, L. (1996). Teacher reports of adjustment to school of postnatally depressed and well mothers' children. (Manuscript submitted for publication.)

Stein, A., Gath, D. H., Bucher, J., Bond, A., Day, A. \& Cooper, P. J. (1991). The relationship between postnatal depression and mother-child interaction. British Journal of Psychiatry 158, 46-52. Teasdale, J. D., Fogarty, S. J. \& Williams, J. M. G. (1980). Speech rate as a measure of short term variation in depression. British Journal of Social and Clinical Psychology 19, 271-278.

Teti, D. M., Gelfand, D. M., Messinger, D. S. \& Isabella, R. (1995).
Maternal depression and the quality of early attachment: an examination of infants, preschoolers, and their mothers. Developmental Psychology 31, 364-376.

Weissman, M. M. \& Paykel, E. S. (1974). The Depressed Woman: A Study of Social Relationships. University of Chicago Press: Chicago.

Whiffen, V. E. \& Gotlib, I. H. (1989). Infants of postpartum depressed mothers: temperament and cognitive status. Journal of Abnormal Psychology 98, 274-279.

Wrate, R. M., Rooney, A. C., Thomas, P. F. \& Cox, J. L. (1985). Postnatal depression and child development: a three-year followup study. British Journal of Psychiatry 146, 622-627. 